\title{
3-D kinematics in low foreground extinction windows of the Galactic bulge $\dagger$
}

\author{
M. Soto ${ }^{1}$, K.Kuijken ${ }^{1}$ and R.M. Rich ${ }^{2}$ \\ ${ }^{1}$ Leiden Observatory, Leiden University, PO Box 9513, 2300RA Leiden, The Netherlands \\ email: soto@strw.leidenuniv.nl \\ ${ }^{2}$ Dept. of Physics and Astronomy, University of California, Los Angeles CA 90095-1562, U.S.A.
}

\begin{abstract}
We report the progress in our measurement of the 3-D kinematics in several low foreground extinction windows of the Galactic Bulge. In order to complete the 3-D kinematics of as many stars as possible, proper motions and radial velocities have been obtained using HST WFPC2-ACS and VLT VIMOS respectively. Our preliminary results for fields close to the galactic minor axis show a significant vertex deviation, which can be directly related to a signature of triaxility. This is one of the strongest proofs to date of the presence of the stellar bar in the galactic bulge.
\end{abstract}

Keywords. Galaxy: bulge, Galaxy: kinematics and dynamics, techniques: radial velocities

\section{Introduction}

The fact that we still lack a unified detailed picture for our galactic bulge is explained by several reasons. Firstly, high foreground extinction towards the bulge, which is uneven even in small scales, limits the optical work to small "windows" where the extinction is known to be moderate. Secondly, even in those cases where the extinction is relatively small, a high contamination by disk populations is expected. This contamination is particularly difficult to identify just based in the CMD, due to the overlapping of bulge and disk populations in several regions of the CMD as the turn-off; thus more elaborated selection criteria are needed.

In spite of all these complications remarkable advancements have been achieved over the years. Spaenhauer et al. (1992) was the first in measuring proper motions for a significant sample of bulge stars using plates with a time baseline over 30 years. The latter study was the starting point for several subsequent projects which measured the abundances and radial velocities of the original Spaenhauer sample (e.g. Terndrup et al. 1995, Sadler et al. 1996). Zhao et al. (1994) used a subsample of the Spaenhauer stars, with metallicities and radial velocities by Rich $(1988,1990)$, to model the kinematics of stars of the galactic bulge; showing that in spite of his small sample size the kinematics features observed were consistent with the presence of a stellar bar. Such a bar signature is in agreement with the results of Binney et al. (1991) for molecular gas, and those from the COBE satellite (Dwek et al. 1995) which show an asymmetry consistent with a bar with its closest end in the first galactic quadrant. These asymmetries have been reproduced for barred models of the Milky Way (Zhao 1996, Häfner et al. 2000, Englmaier and Gerhard 1999).

This project is about measuring the 3 -D kinematics of stars of the galactic bulge in

$\dagger$ Based on observations made with the NASA/ESA Hubble Space Telescope and obtained at the Space Telescope Science Institute, proposals GO-8250 and GO-9816; and on observations made with the European Southern Observatory Very Large Telescope, Paranal, Chile, program 71.B-3048(A) 

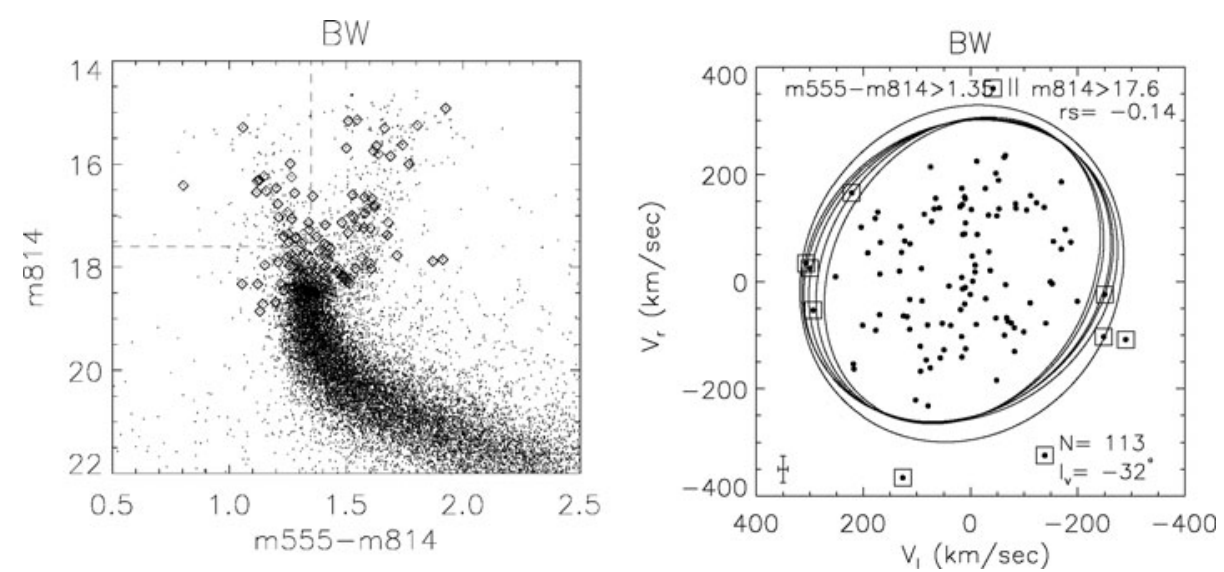

Figure 1. Left, Color Magnitude diagram for one of our fields, Baade's Window. Black points correspond to stars with proper motion information and those enclosed in grey squares have radial velocities as well. Bright blue main sequence stars (foreground; disky kinematics) above the turn-off have been excluded (dashed line) from the final bulge star sample. Right, velocity ellipsoid for radial velocities and transversal proper motions. Here $l_{v}$ is the vertex angle and $\mathrm{r}_{S}$ corresponds to the Spearman rank order correlation coefficient. An iterative clipping algorithm has been used to reject stars beyond $2.5 \sigma$ in the velocity ellipsoid in order to avoid contamination.

order to constrain its phase-space distribution function. The understanding of kinematics of a significant stellar population, can be the key to the understanding of processes responsible of the bulge formation and evolution.

\section{Proper Motions \& Radial Velocities}

Our project includes the observation of several fields across the bulge (Kuijken 2004); where the results showed here correspond to the three first fields close to the galactic minor axis Sagittarius-I $(l, b)=(1.26,-2.65)$, Baade's Window $(l, b)=(1.13,-3.76)$ and near NGC6558 $(l, b)=(0.28,-6.17)$.

The proper motion procedure is a modification of the Anderson \& King (2000) approach, which basically consists of a PSF reconstruction and PSF core fitting using bright stars on the HST images (Kuijken \& Rich 2002). Second epoch observations over a baseline of 6 years have allowed us to accomplish accuracies better than 1 mas $\mathrm{yr}^{-1}$.

Similarly, the radial velocity technique has been developed to work in crowded fields taking advantage of the positions and magnitudes derived from the proper motion procedure. In order to optimize the extraction of star spectra from the VLT VIMOS IFU data-cube, we have combined the IFU PSF and HST positions and magnitudes to separate the contributions of each star from the flux of each fiber in a deconvolution. Blending and overlapping are controlled using HST coordinates to generate convolved images, thus only stars that contribute in a significant amount to the flux of the fiber in which they lay are selected for the radial velocity measurement.

\section{Measurements}

Figure 1 illustrates the detection of radial velocities in one of our fields, close to the galactic minor axis, over the total proper motion measurements. Typically, 200 stars were finally selected per field to have a radial velocity value. Nevertheless, a second 

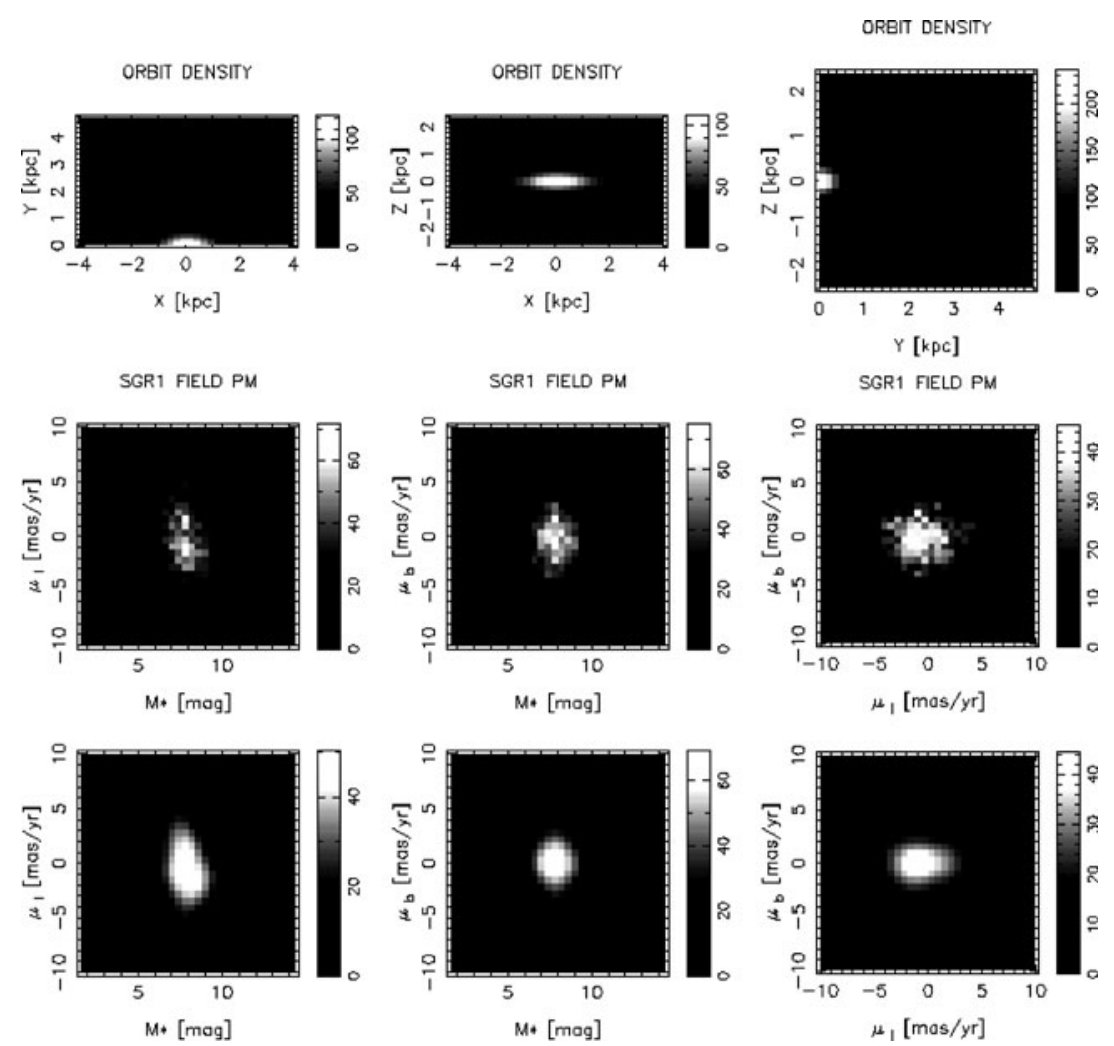

Figure 2. Example of best fit of our model in one of our fields (Sagittarius-I). The first row corresponds to the input density distribution of the bar, where the second and the third are the input and best fit distribution of photometric parallax $\left(\mathrm{M}^{*}\right)$ and the proper motions $\mu_{l}$ and $\mu_{b}$. The orbit library contained 1000 orbits, for a bar pattern speed of $40 \mathrm{~km} \mathrm{sec}^{-1} \mathrm{kpc}^{-1}$, a bar angle of $20^{\circ}$ and a bar mass of $1.2 \times 10^{10} M_{\odot}$.

selection criterion has been applied in order to select bulge stars. CMD combined with the proper motion information show that the kinematics of bright blue main sequence stars is consistent with the kinematics of a disk population rotating in front of the bulge (Kuijken \& Rich 2002). Consequently, our final selection (for this preliminary subsample) of bulge stars with 3-D kinematics excludes those bright blue stars over the turn-off in the CMD.

Velocity ellipsoids have been constructed for fields close to the galactic minor axis (figure 1). The vertex angle $l_{v}$, which corresponds to the angle between the major axis of the velocity ellipsoid and the line of sight (Zhao et al. 1994), is a sensitive probe of deviations of the potential from an axisymmetry. Such is the case of our velocity ellipsoids, where we have found a significant vertex deviation for at least two of the central fields on this project. This signature of triaxility is in agreement with the results of $300 \mathrm{~K}$ Giants in Baade's Window with well defined 3-D kinematics and metallicities (Soto et al. 2007). The latter sample presents a strong difference in the kinematics around - 0.5 dex, with a significant vertex deviation for the metal rich population in comparison to the metal poor one.

Thus, all evidence in these studies points to a triaxial bulge, where contamination of the samples by disk population increases for fields close to the galactic plane. This should be one of the strongest demonstrations to date of the presence of the stellar bar. 


\section{Model}

Our models are based on the Schwarzschild technique (Schwarzschild 1979) which tries to reproduce the full density distribution of the bar plus the distribution of the two proper motions $\left(\mu_{l}, \mu_{b}\right)$ and a photometric parallax $\mathrm{M}^{\star}$ (Kuijken 2004). A subsample of MS and turn-off stars are used to build the 3-D grids which are fitted in several fields simultaneously.

Consequently, the input density of the model is constructed from an analytic deprojection of the COBE map with several free parameters. The latter is used in turn to build a consistent potential with an eight order multipolar expansion (Englmaier and Gerhard 1999).

Through these models we expect to determine bar parameters such as pattern speed, bar angle and bar mass. The extent of our fields across the bulge will allow us to determine fundamental parameters poorly constrained to date.

\section{Conclusions}

Our bulge seems to be triaxial; in agreement with previous evidence it shows asymmetries consistent with the presence of a stellar bar. Vertex deviation clearly appears in our fields and it is even reproduced in our preliminary models. Data and further fields at higher longitudes will further constrain the model allowing us unveil a more detailed picture of our galactic bulge.

\section{Acknowledgements}

MS would like to acknowledge the travel support from Leids Kerkhoven-Bosscha Fonds. RMR acknowledges financial support from HST GO-9816, the Space Telescope Science Institute.

\section{References}

Anderson, J. \& King, I. R. 2000, PASP, 112, 1360

Binney, J., Gerhard, O. E., Stark, A. A., Bally, J., \& Uchida, K. I. 1991, MNRAS, 252, 210

Dwek, E., et al. 1995, ApJ 445, 716

Englmaier, P. \& Gerhard, O. 1999, MNRAS, 304, 512

Häfner, R., Evans, N. W., Dehnen, W., \& Binney, J. 2000, MNRAS, 314, 433

Kuijken, K. \& Rich, R. M. 2002, AJ, 124, 2054

Kuijken, K. 2004, $A S P C, 317,310 \mathrm{~K}$

Rich, R. M. 1988, $A J, 95,828$

Rich, R. M. 1990, ApJ, 362, 604

Sadler, E. M., Rich, R. M., \& Terndrup, D. M. 1996, AJ, 112171

Schwarzschild, M. 1979, ApJ, 232, 236

Spaenhauer, A., Jones, B. F., \& Withford, E. 1992, AJ, 103, 297

Soto, M., Rich, R. M., \& Kuijken, K. 2006, astro-ph/0611433

Terndrup, D. M., Rich, R. M., \& Sadler, E. M., 1995, AJ, 110, 1774

Zhao, H. S., Spergel, D. N., \& Rich, R. M. 1994, AJ, 108, 2154

Zhao, H. S. 1996, MNRAS, 283, 149 